\title{
ISSUES OF TIME FOR REFERENCE SYSTEMS
}

\author{
P. K. Seidelmann \\ U.S. Naval Observatory \\ 3450 Massachusetts Ave., N.W. \\ Washington, DC 20392-5420
}

\begin{abstract}
At the International Astronomical Union General Assembly in 1991 the terminologies Geocentric Coordinate Time (TCG) and Barycentric Coordinate Time (TCB) were introduced as the time coordinates of four dimensional geocentric and barycentric coordinate systems, respectively. The reasons for these time-like arguments and their applications will be discussed. The relationships between the different time-like arguments will be discussed along with their applications and relationships to the astronomical constants.

Since these time-like arguments have secular differences, there are new issues to be resolved. These new issues include the definition of the epoch J2000.0, relationship between mean and apparent sidereal time, the time-like argument for specifying constants, the definition and future use of ephemeris time, delta $\mathrm{T}$ values and the transition to, or future use of, TCG, TCB and TDB.
\end{abstract}

\section{INTRODUCTION}

Beginning in 1984, the time scales Terrestrial Dynamical Time (TDT) and Barycentric Dynamical Time (TDB) were introduced (IAU Transactions, 1977). During the 1980s it was recognized that there were some technical and perceptual problems with these two timelike arguments (Guinot and Seidelmann, 1988). As a result, the IAU Working Group on Reference Systems, (Hughes et al, 1991) included in the nine recommendations, which were adopted by the IAU in 1991, recommendations for the introduction of Geocentric Coordinate Time (TCG) and Barycentric Coordinate Time (TCB) and the renaming of Terrestrial Dynamical Time (TDT) as Terrestrial Time (TT). The relationships between these different time scales and the reasons for their introduction were given by Seidelmann and Fukushima (1992). The recommendations adopted in 1991 by the IAU included the establishment of a Working Group on Astronomical Standards (WGAS) to prepare a draft system of astronomical units and constants, a list of best estimates of astronomical quantities, and standard procedures for fundamental astronomy in the form of tested 
software. This Working Group has established four subcommittees to carry out their work; one of these subcommittees being the subcommittee on Issues on Time, of which this author is the chairman.

\section{J2000.0 DEFINITION}

The epoch J2000.0 has been defined to be the epoch of 2000 January $1.5=$ Julian Date 2451545.0 TDB. This has been specified as the epoch of the FK5 star catalog and used as the epoch of the DE200/LE200 ephemerides. With the secular differences between TCB, TCG, and TT, there are significant differences between the time scales at the epoch J2000.0, as shown by the following values:

$$
\begin{aligned}
& \text { TDT }=\text { TT }=\text { TDB }+0 . .^{\mathrm{s}} 0000994 \ldots-\mathrm{v}_{\mathrm{e}} \cdot\left(\mathrm{x}-\mathrm{x}_{\mathrm{e}}\right) / \mathrm{c}^{2} \\
& \mathrm{TCB}=\mathrm{TDB}+11 . \mathrm{s}^{\mathrm{s}} 25362 \ldots \\
& \mathrm{TCG}=\mathrm{TDB}+0 . \mathrm{s}^{\mathrm{s}} 5057339 \ldots-\mathrm{v}_{\mathrm{e}} \cdot\left(\mathrm{x}-\mathrm{x}_{\mathrm{e}}\right) / \mathrm{c}^{2}-\mathrm{P} \\
& \mathrm{TCG}=\mathrm{TT}+0 . .^{\mathrm{s}} 505833 \ldots \\
& \mathrm{TCB}=\mathrm{TCG}+10 .{ }^{\mathrm{s}} 74778 \ldots+\mathrm{v}_{\mathrm{e}} \cdot\left(\mathrm{x}-\mathrm{x}_{\mathrm{e}}\right) / \mathrm{c}^{2}+\mathrm{P} \\
& \mathrm{UT} 1 \approx \mathrm{TDB}-65 .^{\mathrm{s}}
\end{aligned}
$$

where $x_{e}$ and $v_{e}$ denote the barycentric position and velocity of the Earth's center of mass at $\mathrm{J} 2000.0, \mathrm{x}$ denotes the barycentric position of the observer, $\mathrm{c}$ is the speed of light, and $\mathrm{P}$ are periodic terms, whose largest amplitude is 1.6 millisecond.

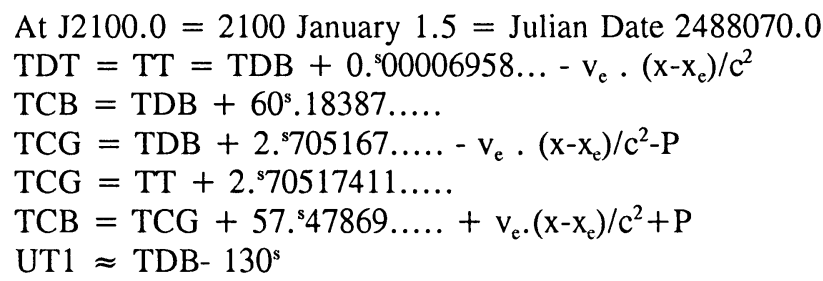

So we have a choice to make in the designation of the epochs J2000.0 and J2100.0. We can choose to retain the concept of the epoch being a fixed event, i.e. the specified time in $\mathrm{TDB}$, or TT, and then determine the equivalent time for that event in the different time scales. Alternatively, we can choose to have J2000.0 be JD2451545.0 in all time scales. Thus, the epoch will be different depending on the time scales. Specifically, in the TT time scale there will be different times for the J2000.0 epoch,as defined in the TCB, TCG, TDB and TT time-like arguments.

The most logical proposal to date is to define the J2000.0 epoch in TT. This results in an insignificant change from the definition in TDB. It is in terms of one of the "new" time scales, yet retains a continuity with the older time scales and the previous definition.

\section{Julian Century}

There is a corresponding issue for the definition of the Julian Century and its use for star catalogs and astrometric constants. The Julian Century could be defined as 36525 days in 
one time scale, i.e. in TT, in which case the length would be slightly different in other time-like arguments, such as TCB and TCG. Alternatively, the Julian Century can be defined as 36525 days of time in all time-like arguments, in which case for any one timelike argument there will be a set of equivalent values of the Julian century defined for the other time-like arguments.

Again, it has been suggested that the Julian century be defined as 36525 days of TT. This would be consistent with the definition of the epoch. Then a Julian Century of 36525 days of TT implies an equivalent time period in the other time-like arguments of:

$$
\begin{array}{ll}
\text { TCG } & 36525^{\mathrm{d}}+2 .^{\mathrm{s}} 199340787 \ldots=36525^{\mathrm{d}} .00002546 \\
\text { TCB } & 36525^{\mathrm{d}}+48 .^{\mathrm{s}} 93024815 \ldots=36525^{\mathrm{d}} .00056632
\end{array}
$$

\section{Relationship Between Mean and Apparent Sidereal Time}

The International Earth Rotation Service (IERS) has proposed a change in the relationship between Greenwich Mean Sidereal Time (GMST) and Greenwich Apparent Sidereal Time (GAST). Woolard and Clemence (1966) give the expression for the rotation angle of the Earth measured from the true equinox. Aoki and Kinoshita (1983) examine the relationship between the non rotating origin and the equinox and develop the true expression. Based on their derivation the new recommended expression is:

$$
\begin{gathered}
\mathrm{GAST}=\mathrm{GMST}+\Delta \psi \cos \epsilon_{0}+0 . " 00264 \sin \Omega \\
+0 . " 000063 \sin 2 \Omega
\end{gathered}
$$

which adds two terms dependent upon the longitude of the lunar node $(\Omega)$ to the term for the "equation of the equinoxes" which is based on the nutation in longitude $(\Delta \psi)$ and the mean obliquity of epoch $\left(\epsilon_{0}\right)$. The above expression includes all the periodic terms given by Aoki and Kinoshita (1983) for the accumulated precession and nutation in right ascension. The effects of these terms on the estimation of UT1 have been described by Gontier and Capitaine (1991). The expression can be interpreted as redefining the "equation of the equinoxes," if you consider that as the difference between the time scales. However, the equation of the equinoxes is really defined in geometric terms.

These terms are being introduced because they are now detectable in observations taken for determining Sidereal Time, while in the past they were too small to be detected. The suggestion is to introduce these terms on February 26, 1997, at the time when their value is zero so there would be no discontinuity in Sidereal time and UT1. The IERS Board has recommended the inclusion of these terms and the IAU should consider whether to recommend this change also.

\section{EPHEMERIS TIME}

There is a practical difficulty with our definition of a time scale prior to 1956 . We have defined TDT or TT in terms of TAI, but there is no TAI prior to 1956. The definition of TDT prior to the availability of TAI was not specified and was left to be determined from the ephemerides according to the definition of ephemeris time. The epoch of ephemeris time is specified in terms of the geometric mean longitude of the Sun under the old system 
of constants with Newcomb's Theory of the Sun. However, geometric mean longitude of the Sun means there is an expression for the mean longitude of the Sun to which perturbations and aberration will be added to obtain the apparent position. With numerical integrations there is no such expression and for general theories the expression depends on the long-period perturbation terms.

The epoch is defined for 1900 but continuity with TDB and TDT is desired at the epoch 1955 when they can be determined.

The definition of the ephemeris second is a fraction of the tropical year for 1900, which is defined as the period of the Sun's motion from equinox to equinox. The conventional value of precession for 1900.0 was $5025^{\prime \prime} .73976 /$ century, but the new IAU value of precession introduced in 1984 is 5026".87817/ century, a difference of $1 " .13441 /$ century. The Sun moves 0".04106705/ephemeris second. The IAU (1976) change in the constant of precession then implies an increase in the tropical year of $0^{\mathrm{s}} .276234$.

It seems desirable to redefine Ephemeris Time for times prior to the availability of atomic time in terms of the SI second. One disadvantage is that the unit of time will not be directly derivable from the ephemerides. The ephemeris time revised $\left(\mathrm{ET}_{\mathrm{R}}\right)$, for use as a time scale prior to the availability of TAI or AT, could be defined as follows:

(a) Ephemeris time revised $\left(\mathrm{ET}_{\mathrm{R}}\right)$ is reckoned from the instant $1958 \mathrm{Jan} 0^{\mathrm{d}} 0^{\mathrm{h}} \mathrm{TAI}$ at which time $\mathrm{ET}_{\mathrm{R}}$ has the value $1958 \mathrm{Jan} 0^{\mathrm{d}} 0^{\mathrm{h}} 0^{\mathrm{m}} 32^{\mathrm{s}} .184$, and

(b) The unit of time of $\mathrm{ET}_{\mathrm{R}}$ is the SI second.

The alternative argument to revising the definition of Ephemeris Time is that the current definition of Terrestrial Time implicitly is the same definition of a time scale prior to 1956, as the explicit definition given by this revised ephemeris time.

\section{DELTA T TABLES}

The values of Delta T (ET-UT) are dependent upon the ephemerides, the value of the secular acceleration of the Moon, the reference system, and the constants, such as precession. This dependence varies according to the methods used to determine Delta $T$. The values of Delta $T$, which were determined based on one set of constants, ephemerides, and reference system, cannot accurately be used with a different reference system. To illustrate the dependence upon ephemerides, the following comparisons for the lunar and solar mean motions are tabulated. A change of 1 "/century in the Sun's mean motion means a change of $24 .{ }^{5} 35$ per century in the values of delta $T$. Also a change of 1 "/century in the Moon's mean motion implies a change of $1 .{ }^{s} 8214$ per century in the values of delta $\mathrm{T}$.

The lunar ephemeris of Chapront-Touze and Chapront (CTC) (1988), has been fit to the LE200 ephemeris. CTC differs from the Improved Lunar Ephemeris, which has been the basis of most previous delta $T$ determinations, in the mean motion as tabulated. This results in the differences of delta $\mathrm{T}$ at the epochs given in Table 1

A similar comparison between the Solar mean motions, as given by Bretagnon and Simon (1991) and by Newcomb (1895), gives almost constant differences of one arcsecond per century, which implies a change of about $26^{\mathrm{s}}$ per century in delta $\mathrm{T}$ values.

The values of delta T from 700BC to AD1980 have been given by Stephenson and Morrison (1984). The determinations are primarily from Solar eclipse and occultation 
observations. The changes in delta $\mathrm{T}$ will depend on the type of observation. There is also the complication that the tidal acceleration of the mean longitude of the Moon must be assumed, or determined from other sources, to separate it from the Delta $\mathrm{T}$ determination.

Table 1 Differences in Lunar Mean Motion and Delta T

$\begin{array}{ccl}\text { Epoch } & \text { CTC -ILE } & \text { Resulting change } \\ & \begin{array}{l}\text { Mean motion Diff } \\ \text { " per century }\end{array} & \begin{array}{l}\text { in Delta T } \\ \text { s per century }\end{array} \\ 2000 & 1 . " 66 & 3^{\mathrm{s}} .02 \\ 1900 & 3.10 & 5.65 \\ 1800 & 4.53 & 8.25 \\ 0 & 31.95 & 58.19\end{array}$

\section{OTHER ISSUES}

There are two issues that keep coming up for consideration, but each time the status quo seems to be better than any alternative solutions. One of these issues is the 32.184 second difference between Terrestrial Time and TAI. There is strong opposition to a change in either of the two timescales which would be necessary to eliminate the difference. The other problem is the difficulty and confusion due to the requirement to introduce leap seconds into the UTC time scale. The alternative would be to permit a secular increase in the difference between UTC and UT1. If, at some time in the future, the decision were made not to continue to introduce leap seconds in UTC, it might be advisable to introduce steps such that UTC, TAI, and TT are equal.

\section{TCG AND TCB IMPLEMENTATION}

The resolutions adopted by the IAU in 1991 recommended the use of TCG and TCB, and authorized the continued use of TDB. There are strong opinions concerning the planned use, or lack of use, for these different time like arguments by different organizations. Many of the issues of time have been raised through the circulars of the Astronomical Standards Working Group. The responses have been quite limited. I do not know if the limited response is a lack of interest, a lack of understanding, a wait-and-see attitude, a desire to ignore the issues in favor of the status quo, or a feeling that there is no urgency to consider the issues.

Therefore, I will propose a straw-man recommendation for consideration. I would recommend that 1) Terrestrial Time (TT), previously called Terrestrial Dynamical Time (TDT), be accepted as the common standard of time for the definition of the J2000.0 Reference System and for the Julian Century, and be the basis for conversion to the other time units. Thereby, organizations so choosing can continue to use TDB while others can choose TCG and TCB. 2) The Astronomical Standards Working Group provide values for constants with specific indications of the time scale used for the constants. Thereby, the constants can be converted to other time scales according to their needs and uses. 3) Based on the new constants and definitions, new ephemerides should be developed with solutions for quantities, recognizing their dependence upon the time scales used. 4) The revised 
relationship between mean and apparent Sidereal Time should be adopted for introduction in 1997 as recommended by the IERS. 5) The revised definition of ephemeris time should be adopted, so that the time-like argument for use of ephemerides prior to 1956 is clear, and so that the implications are better understood.

\section{SUMMARY}

The concepts of TT, TCB, and TCG have been introduced by the IAU to meet the requirements for more accurate time-like arguments, defined in strict conformity with the theory of relativity. They provide a better standardized terminology in accordance with requirements for increased accuracy. Over the past 40 years, the progress in standards has been to eliminate ambiguities in standards definitions and to improve the accuracy levels. This progression involving astronomical quantities, ephemerides, reference systems, star catalogs, and time-like arguments will logically and hopefully continue.

\section{References}

Aoki, S., and Kinoshita, H., (1983) "Note on the Relation Between the Equinox and Guinot's Non-rotating Origin," Celest. Mech. 29, 335-360.

Bretagnon, P., and Simon, J.L., (1991) private communication.

Chapront-Touze, M. and Chapront, J. (1988) "ELP 2000-85; a Semi-analytical Lunar Ephemeris Adequate for Historical Times" Astron. Astrophys. 190, 342-352.

Comite International des Poids et Measures (1957), Proces Verbaux des Seances, deuxieme serie 25, 75-79.

Gontier, A.M. and Capitaine, N. (1991) "High Accuracy Equation of Equinoxes and VLBI Astrometric Modelling" Astronomical Society of the Pacific Conference Series, 19, T.J. Cornwell and R.A. Perley eds, pp 342-345.

Guinot, B. and Seidelmann, P.K. (1988) "Time Scales: their history, definition and interpretation" Astron. Astrophys 194, 304-308.

Hughes, J.A., Smith, C.A., and Kaplan, G.H., (1991) IAU Proceedings of the 127th Colloquium of the IAU, Reference Systems, U S Naval Observatory, Washington, DC.

Newcomb, S. (1895) "Tables of the Motion of the Earth on its axis Around the Sun," Astron Papers Amer Ephem VI, I.

Seidelmann, P.K. and Fukushima, T. (1992) "Why New Time Scales?" Astron. Astrophys. in press.

Stephenson, F.R. and Morrison, L.V. (1984) "Long-term Changes in the Rotation of the Earth: 700 BC to AD 1980" Phil Trans R. Soc London A313, 47-70.

Woolard, E.W., and Clemence, G.M. (1966) Spherical Astronomy, Academic Press, New York and London.

(1977) Proceedings of the Sixteenth General Assembly, Transactions of the IAU, XVI, B. D. Reidel Publishing Co., Dordrecht.

(1992) Proceedings of the Twenty-First General Assembly, Transactions of the IAU, XXI B Kluwer Academic Publishers, Dordrecht. 